\title{
Guilleermo
}

de Delcham

Editorial ə Acceso abierto

\section{Sistemas dinámicos y pandemias. Una relación para pensar mejor}

\section{Dynamic systems and pandemics. A relationship to think better}

\author{
Rebeca Puche Navarro, ii \\ Ángela González iii \\ i. Coporación Niñez y Conocimiento; Cali; Colombia. \\ ii. Editorial California-edit; Bogotá; Colombia. \\ iii. Investigadora independiente; Bogotá; Colombia.
}

Correspondencia. Rebeca Puche Navarro, Email: direccion@california-edit.com

Citar así: Puche-Navarro, Rebeca; González, Ángela. (2021). Sistemas dinámicos y pandemias. Una relación para pensar mejor. Revista Guillermo de Ockham. 19(1), 3-6.

https://doi.org/10.21500/22563202.5389

Copyright: () 2021. Universidad de San Buenaventura, Cali. La Revista Guillermo de Ockham proporciona acceso abierto a todo su contenido bajo los términos de la licencia Creative Commons AttributionNonCommercial-NoDerivatives 4.0 International (CC BY-NC-ND 4.0).

Conflicto de intereses. Los autores declaran que no hay conflicto de intereses.

Disponibilidad de datos. Todos los datos relevantes están en el artículo. Para mayor información contactar al autor de correspondencia.

Descargo de responsabilidad. El contenido de este artículo es responsabilidad exclusiva de los autores y no representa una opinión oficial de sus instituciones ni de la Revista Guillermo de Ockham.
El término complejidad empieza a encontrarse prolijamente en la literatura académica a partir de la década de 1970 y hoy en día es un sello de pensamiento sofisticado. Los sistemas dinámicos no lineales son una de las caras del estudio de los fenómenos complejos y aunque su historia rebasa el siglo XX, se considera que solamente en ese siglo se consolidan y constituyen, lo que algunos no vacilan en considerar un nuevo paradigma (Morin, 2009; Munné, 2013). Lo cierto es que desde una dinámica no lineal, se abordan fenómenos que a partir de visiones convencionales mostraban ser reacios a entenderse de manera más completa. Según ese paradigma, por ejemplo, se puede advertir que para muchos fenómenos las consecuencias no son proporcionales a las causas y que los puntos de llegada no tienen que ver con el punto de partida (Prigogyne, 1993). En resumen, introducen una lógica basada en parámetros críticos que nos hacen repensar.

La dimensión temporal (léase dinámica), juega un papel privilegiado y ella se combina con procesos en los que priman la interacción entre múltiples elementos interactuantes (Espinoza, 2003). De esas interacciones que evolucionan en el tiempo, emergen consecuencias no lineales. Recuérdese que con las relaciones lineales hay que separar el todo en partes para resolverlas y luego proceder a juntar las soluciones, algo no siempre posible ni deseable cuando el todo es mucho más que la suma de sus partes. Desde la gestalt sabemos que cuatro elementos no son la suma de ellos, pues dependen de la estructura en la que estén dispuestos (y en la que pueden conforman un cuadrado, entre otras figuras).

La dificultad en aceptar pensar de manera distinta a lo que las concepciones dominantes nos han educado, quizás explique la tardanza en adoptar esa dinámica no lineal de manera más amplia.

Sabemos que en la naturaleza proliferan los fenómenos no lineales, aquellos que no siempre responden a una relación causa-efecto de manera directa, proporcional y secuencial (Mandelbrot, 2002). Ese análisis no lineal puede ser, sin embargo, especialmente pertinente en el momento de mejorar la comprensión del mundo en que vivimos. Las relaciones humanas ejemplifican fenómenos complejos no susceptibles de entenderse desde una lógica simple. Ocurre lo mismo con otros, como el tiempo atmosférico. Lorenz demostró que pequeñas variaciones en los inicios de los itinerarios pueden tener vastas repercusiones imprevisibles a 
lo largo del tiempo. ${ }^{1}$ Igualmente ilustrativa es la imagen de Mandelbrot: "Las nubes no son esferas, las montañas no son conos, las líneas costeras no son círculos, la corteza no es suave, y los rayos no viajan en línea recta". ${ }^{2}$

Las pandemias, por otra parte, son uno de esos fenómenos complejos que a pesar de haber acompañado a la humanidad, siempre sorprenden y parecen desconcertar tanto a dolientes como a gobernantes. Entonces pensar las pandemias desde una dinámica no lineal puede ser iluminante.

Buena parte de los trabajos sobre sistemas complejos remite a los "sistemas dinámicos que tienen como característica central y no anodina poderse representar en ecuaciones diferenciales no lineales" (García, 2000, p. 66. El subrayado en nuestro). A partir de sus tratamientos se pueden construir modelos formales que enriquecen el análisis de los fenómenos estudiados y alcanzan un nivel de comprensión más adecuado.

Un fenómeno que lo ilustra es la interacción no lineal entre dos poblaciones de peces diferenciales que se regulan entre sí el cual es modelizado por ecuaciones y conocido como el depredador-presa de Lotka-Volterra. En 1925, Lotka un ańo antes que Volterra, construye con ecuaciones un modelo que respresenta un ciclo recurrente en el que el aumento de la población de depredadores conduce a la disminución de la población de presas solo hasta un límite fijo, el cual se estabiliza cuando la poblacion de presas es insuficiente para alimentar a los depredadores (Ossa, 2011). Esas interregulaciones que se construyen entre las dos poblaciones, donde la una regula a la otra y viceversa, ilustran los cambios que la relación produce aunque. Aquí las ecuaciones diferenciales se revelan como excelente herramienta.

Lo que resulta en un encuentro inverosímil, es que exactamente en la misma época en que Lotka y Volterra formulan esa ecuacion, dos escoceses Kermack y McKendrick (1927), aplican un modelo que sigue esa lógica (calificado antes de contraintuitiva), respecto a las relaciones de los componentes que conforman ese sistema que es la epidemia. Fue el primer estudio que aplicó modelizaciones provenientes de ecuaciones diferenciales para repensar dos conjuntos que interaccionan con base en relaciones no lineales, no directas ni proporcionales.

En una epidemia, el contagio depende del número de personas con las que un infectado entra en contacto, de los grupos de los que forma parte, de su edad, de su propia historia clínica y de su capacidad inmunológica, entre otras variables. La novedad de Kermack y McKendrick fue tratarla con matemáticas no lineales. Su pregunta inicial era saber el lugar que tiene el agotamiento de los miembros susceptibles de la comunidad en la epidemia. El modelo matemático que idearon -inusual para la época- se basaba en que en todo grupo existe

(...) un umbral de la población en lo que concierne la infectividad, recuperación y tasas de muerte. Si esa densidad de población real es igual (o inferior) a este valor umbral, introducir una o más personas infectadas no da lugar a la epidemia. El tamaño de la epidemia aumenta rápidamente a medida que se excede la densidad umbral y de tal manera que cuanto mayor sea la densidad de población al comienzo de la epidemia, menor será al final de la epidemia (Kermack y McKendrick, 1927, p. 720).

Más que confirmar los hechos, la audacia del modelo ha servido de inspiración y abrió una puerta para dar un nuevo giro a los estudios epidemiológicos. Desde entonces, es un

1. Aquella bella imagen que se hizo célebre y según la cual el aleteo de una mariposa en Singapur podía repercutir en tornados en Texas y al condensarse toma la forma de una mariposa. Tiene uno de los atractores más conocidos. (Para una mayor ilustración ver Puche-Navarro, 2009).

2. Fuente: https://citas.in/frases/2022103-benoit-mandelbrot-las-nubes-no-son-esferas-las-montanas-no-son-cono/ 
hecho que el comportamiento de las pandemias se aborda mediante ecuaciones diferenciales no lineales y con ello se pueden conocer mejor sus implicaciones.

Entre los numerosos estudios de esta tendencia, son muchos los que demuestran con lujo de rigurosidad, que los modelos dinámicos ofrecen información más precisa que los métodos basados en estadísticas (Shoabib et al., 2021). Para esos autores "La influencia de parámetros claves en la propagación de la pandemia de Covid-19 se modelan de manera ideal, lo que ayudará a planificar, monitorear, prevenir y medir la propagación de la pandemia" (p. 14). Otros estudios abordan la relación entre la percepción pública y las políticas gubernamentales para determinar que ellas desempeñan un papel definitivo en la dinámica de la epidemia, y encuentran un patrón de propagación de la evolución de la enfermedad (Kwuimy et al., 2020). Sus muy matizados análisis dinámicos encuentran que “(...) el análisis paramétrico revela condiciones para que ocurra un equilibrio epidémico sostenido" (Kwuimy et al., 2020, p. 11). Otro de los los estudios que muestran la fecundidad del abordaje de la dinámica no lineal de manera exploratoria y libre (como dicen los autores), trabaja "(...) series de tiempo recursivo para estimar los cambios en ciertas métricas que pueden proporcionar información continua sobre el comportamiento de la epidemia"3 (Young y Chen, 2021, p. 1). ${ }^{4}$ Para ellos, “(...) son prometedores los hallazgos del modelado mecanicista basado en datos, usando la estimación de parámetros dependientes". Y agregan: "Ellos sugieren que este enfoque permitiría identificar un modelo epidemiológico no lineal paramétricamente eficiente, comparable con los modelos de simulación no lineal existentes" (Young y Chen, 2021. p. 11).

Este muy sucinto (por falta de espacio) resumen para ilustrar el pensar la pandemia a la luz de métodos derivados de la dinámica no lineal, debe ilustrar los giros inesperados que se pueden encontrar en el camino del conocimiento. Barry (2004), en La gran influenza señala que las epidemias conducen a grandes transformaciones del conocimiento cientifico. Apostarle a una visión de las pandemias desde los sistemas dinámicos, hace avanzar, definitivamente, nuestra manera de pensarlas. El balance es que muchos de los estudios que despegan en esta dirección iniciada con Kermack y McKendrick en el propio siglo XX, conforman actualmente una sólida tendencia (Arch-Tirado y Rosado-Muñoz, 2009; Kwuimy et al., 2020; Shoabib et al., 2021; Young y Chen, 2021, entre otros). Quizás habría que avanzar y hacer más comprensible para todos los públicos los modelos que revelan esa capacidad de pensar la epidemia de otra manera, encontrándole giros inesperados y direcciones no evidentes y que de alguna manera revelan que la capacidad de pensar pensamientos nuevos sigue siendo un camino extraordinariamente frágil y poco frecuentado.

\section{Referencias}

Arch-Tirado, E., Rosado-Muńoz, J. (2009). Ciencias de la complejidad y caos como herramientasen el análisis de la proliferación de vectores y zoonosis. Cirugía y cirujanos, 77(4), 341-350. Recuperado de: https://www.medigraphic.com/cgi-bin/new/resumen.cgi? IDARTICULO=22028

Barry, J. (2004). The great Influenza: The Story of the Deadliest Pandemic in History. Penguin Books UK.

Espinoza, M. (2003). El triste destino del azar. Límite, (10). 1-23.

3. Los Fixed Interval Smoothing (FIS)

4. Los autores invitan a los lectores a utilizar las herramientas "disponibles en el Captain (Caja de herramientas para Matlab), que se puede descargar sin costo desde el sitio web mencionado en el documento" (Young y Chen, 2021. p. 12). 
Kermack, W. O \& McKendirck, A. G. (1927). A Contribution to the Mathematical Theory of Epidemics. Proceedings of the Royal Society A. 115, 700-721. https:/doi.org/10.1098/ rspa.1927.0118

Kwuimy, C.A.K., Nazari, F., Jiao, X., Rohani, P., Nataraj, C. (2020). Nonlinear dynamic analysis of an epidemiological model for COVID-19 including public behavior and government action. Nonlinear Dynamics, 101, 1545-1559. https://doi.org/10.1007/s11071-020-05815-z

Mandelbrot, B. (2002). Fractals, Graphics, and Mathematics Education. En Michael Frame y Benoit Mandelbrot (Eds.). 21-38. Fractals, Graphics, and Mathematics Education. Cambridge University Press.

Morin, E. (2009). Introducción al pensamiento complejo. Gedisa: Madrid

Munné, F. (2013). Perfecto e imperfecto: completo. Estudios sobre la complejidad. California-edit : Bogotá

Ossa, J. C. (2011). Un intextricable juego de pérdidas y ganancias. Acta Colombiana de Psicologia. (14)2. 45-55

Prigogyne, I. (1993). Les lois du chaos. Flammarion: Paris

Puche-Navarro, R. (2009). ¿Es La mente no lineal? Programa Editorial Universidad del Valle: Cali

Shoabib, M., Asif Zahoor Raja, M., Touseef Sabir, M., Hussain Bukhari, A., Alrabaiah, H., Sha, Z., Kuman, P., Islam, S., (2021). A stochastic numerical analysis based on hybrid NAR-RBFs networks nonlinear SITR model for novel COVID-19 dynamics. Computer Methods and Programs in Biomedicine. 202, 1-17. https://doi.org/10.1016/j.cmpb.2021.105973

Young, P. C., \& Chen, F. (2021). Monitoring and forecasting the COVID-19 epidemic in the UK. Annual Reviews in Control. https://doi.org/https://doi.org/10.1016/j.arcontrol.2021.01.004 